\title{
Clean intermittent catheterisation for the neuropathic bladder
}

\author{
A Yadav MBBS MS MCh(Urol), ${ }^{1}$ S Vaidyanathan MBBS MS MCh(Urol) MAMS \\ $\mathrm{PhD},{ }^{1} \mathrm{D}$ Panigrahi MBBS $\mathrm{MD}^{2}$
}

Departments of ${ }^{1}$ Urology and ${ }^{2}$ Microbiology, Postgraduate Institute of Medical Education and Research, Chandigarh-160012, India.

Clean intermittent catheterisation (CIC) has been used as the effective method of bladder drainage in paraplegics for over a decade in our centre. In 27 acute spinal cord injury (SCI) patients managed by $\mathrm{CIC}$ from day one, symptomatic urinary infection occurred in 5 with a follow up period of 10 days to 3 months. No prophylactic/suppressive antibiotics were used. In 21 patients on long term CIC (1-12 years) symptomatic infections occurred during a 6 month follow up at a rate of 0.07 episodes per patient per month. Urine cultures proved positive in $26 \%$. The types of bacteria and their sensitivity towards antibiotics were different in both groups.

\begin{abstract}
Keywords: paraplegia; neuropathic bladder; urinary catheterisation; urinary tract infections.
\end{abstract}

\section{Introduction}

Intermittent catheterisation for the neuropathic bladder has proved to be a very useful method of bladder drainage in the early postinjury period ${ }^{1-3}$ as well as in the later stages after spinal trauma. ${ }^{4}$ The classical study of Lapides et $a l^{5}$ stressed the need for a clean, not necessarily a sterile technique. An important factor is prevention of over-distension of the bladder which compromises the local mucosal defence mechanisms. Many patients on $\mathrm{CIC}$ have positive urine cultures; this does not always mean infection but may indicate contamination or colonisation. Over a period of 6 months we reviewed 21 patients with a neuropathic bladder treated by long term $\mathrm{CIC}$ for $1-12$ years studying the frequency of infective episodes. Also, 27 patients with acute spinal trauma were prospectively studied for short periods during their initial hospitalisation; for 10 days to 3 months, to determine the incidence of urinary tract infection (UTI).

Correspondence: Dr S Vaidyanathan, Registrar in Spinal Injuries, Regional Spinal Injuries Centre, Town Lane, Kew, Southport, Merseyside PR8 6NJ.

\section{Material and methods}

\section{Group A (short term CIC)}

This group $(n=27)$ included acutely injured paraplegics (10) or tetraplegics (17) with a neuropathic bladder, who were followed up until their discharge from hospital. They were admitted 12-96 hours after injury. Delays were in part due to lack of rapid transport facilities as many patients were brought to the hospital in tractors used for agricultural purposes. Also, some patients were required to wait in our hospital emergency area for admission, as no separate spinal trauma facility exists in our hospital and those patients with a SCI had to compete with other surgical emergencies for admission eg patients with acute peritonitis. The minimum period of observation was 10 days and the maximum was 3 months. There were 26 males and one female. CIC was started from the first day of hospitalisation. Patients dying within 10 days were not included in the study. CIC in this group was usually performed by the patient's relatives. An F-12 Foley's catheter was used with a clean (not sterile) technique. No prophylactic antibiotics were used. Urine samples were collected by a sterile technique for a widely microscopic examination and cul- 
ture, but the number of urine culture reports that were available for analysis was much less because the samples did not always reach the laboratory. The test tube containing the urine sample might be broken during transport to the laboratory, and sometimes the sample would be contaminated and the report would be 'Bacterial growth of no significance'. Growth of microorganisms alone was not taken to signify infection. Infections were diagnosed as being clinically significant in the presence of the following criteria, modified from Maynard and Diokno: ${ }^{6}$

(1) purulent urethral discharge with positive urethral culture;

(2) pyuria/foul smelling urine with positive urine culture and white blood cells more than 25/HPF (laboratory infection):

(3) fever of more than $100^{\circ} \mathrm{F} /$ chills/flank pain, with laboratory infection;

(4) increased rigidity/flexor spasms/reflex sweating with laboratory infection;

(5) positive C-reactive protein with positive urine culture.

Such infections were treated with a 7 day course of appropriate antibiotics; asymptomatic bacteriuria was not treated.

\section{Group B (long term CIC)}

The second group of patients $(n=21)$ included 19 paraplegics and 2 tetraplegics. All patients who carried out CIC for a minimum of one year were included in the study. Eight patients were on CIC for more than 6 years; the maximum follow up period was 11 and 12 years respectively in 2 patients. The technique of CIC and the features of the study were the same as was carried out in group A, except that the urine cultures were done 3-monthly or whenever the patient was symptomatic. Patients with positive cultures were asked for a repeat sample of urine and if the same organism was grown along with pus cells of more than 25/HPF on microscopy, this was taken as evidence of urinary infection in symptomatic patients. A few patients received pharmacological agents acting on the bladder, such as probanthine, oxybutynin, etc, in addition to the treatment by CIC.

\section{Results}

\section{Group A}

Out of 27 patients, $5(18.5 \%)$ had a clinically significant infection during their initial hospitalisation (urethral discharge/urethritis: 3; pyuria: 3; fever: 5; flexor spasms: 2 ). There was a high incidence of positive urine cultures. Out of a total of 41 cultures obtained in 27 patients, $28(68 \%)$ grew various organisms (Fig 1). Only 13 of the 41 urine samples were sterile. These organisms were mostly resistant to common antibacterial drugs, such as cotrimoxazole, nalidixic acid and gentamicin. None of these patients developed a serious urological complication such as septicaemia or a lower urinary tract complication.

\section{Group B}

During a 6 month follow up 7 out of 21 patients $(33 \%)$ on long term CIC showed evidence of recent clinical infection manifested by fever, pyuria, and flank pain; and associated with or without incontinence of urine. One patient had two episodes of infection in the last 6 months of follow up, thus there were eight infective episodes in the 21 patients. The frequency of infection per patient per month was 0.07 (9 episodes $/ 6$ months $\times 21$ patients). The number of patients exhibiting positive urine cultures (laboratory infection) during the same period was $16(76.2 \%)$. Positive cultures in asymptomatic patients were ignored while five vaguely symptomatic (eg doubtful history of pyrexia, urethral pain etc) cases were tested by C-reactive protein which proved positive in only one patient. Thus, 8 patients had nine episodes of infection in 6 months.

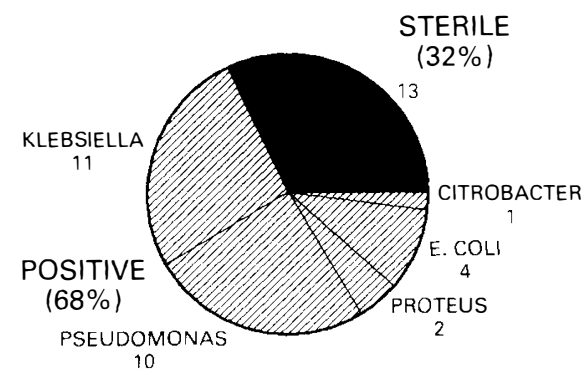

Figure 1 Group A urine samples $(n=41)$. 
Asymptomatic patients with positive cultures were observed, had no treatment and none developed an acute UTI or upper urinary tract complications.

The organisms grown in group B patients were distinctly different from those in group A (Fig 2). Another dissimilarity was that these organisms were often sensitive to drugs such as cotrimoxazole, gentamicin and norfloxacin.

\section{Discussion}

Urinary complications, especially sepsis, are the commonest cause of death in patients after spinal cord injury in the long term in our centre, in contrast to the situation in spinal units in Western countries. We have a significant number of paraplegic patients with a serum creatinine level of $\simeq 8 \mathrm{mg} \%$. Thus we continue to see SCI patients with renal failure from urinary complications.

There are various methods of treating the neuropathic bladder in SCI patients, including an indwelling catheter, a condom catheter, a suprapubic catheter; and catheter-free methods such as reflex voiding and Credé's manoeuvre. Each of these has its own hazards; but clean self intermittent catheterisation has emerged as the method of choice in recent years. ${ }^{7-9}$ The commonest reservation against CIC expressed by many patients and unfortunately also by some physicians concerns the possible risk of introducing infection. In our study, we found that positive urine cultures were common but did not indicate clinically significant infection in the absence of other criteria, and hence did not indicate the necessity for therapy. Mohler et al ${ }^{10}$ com-

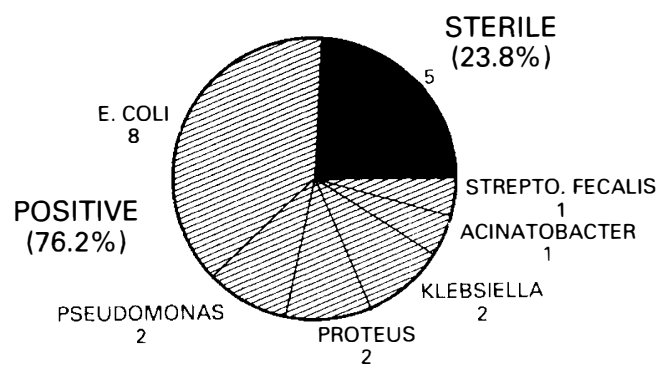

Figure 2 Group B urine cultures $(n=21)$. pared suppressive therapy with trimethoprim-sulfamethoxazole to placebo and found no difference in the rates of symptomatic or total urinary tract infection. They also showed that the treatment of asymptomatic UTI, a full course of antibiotics (10 days), offered no advantage over the treatment of symptomatic infection only, a short course (3 days) of therapy, in management of patients with a neuropathic bladder on CIC. Maynard and Diokno ${ }^{6}$ showed in 50 patients that antibacterial prophylaxis significantly reduced the probability of 'laboratory', but not of 'clinical' infection. Dean ${ }^{11}$ on the other hand, failed to demonstrate any significant difference in the incidence of bacteriuria whether there was no prophylaxis, or using cotrimoxazole followed by nitrofurantoin. In our study, the clinical infection rate on short term CIC was lower $(18.5 \%)$ than the expected rates for patients with an indwelling catheter, and comparable to the rate $(26 \%)$ reported by Maynard and Diokno. ${ }^{6}$

Thirteen $(23.8 \%)$ of our patients maintained a sterile urine at the time of discharge from hospital, compared to the series of Maynard and Diokno ${ }^{6}$ : $12 \%$; Ott and Rosier $^{3}$ : 31\% ; Pearman ${ }^{12}$ : 50\% ; and Donovan et $a l^{13}: 23 \%$. These infections, if promptly treated, did not cause serious complications. In our series, no patient developed upper tract deterioration or vesicoureteral reflux (VUR) on long term follow up. Upper tract damage is known to occur in these patients if CIC is performed infrequently, thus allowing the bladder pressure to rise, or if VUR is present. ${ }^{14}$

The microorganisms obtained on urine culture in our series are strikingly different in groups A and B. While Klebsiella pneumoniae and Pseudomonas aeruginosa were responsible for $75 \%$ of infections in short term CIC patients, the same organisms were grown in only $25 \%$ of long term follow up cases. Similarly, E. coli was grown from $14 \%$ of group A cultures as compared to $50 \%$ of group B. This difference can be explained by the fact that Klebsiella and Pseudomonas are acquired by the patient initially whilst in the hospital, but later the patient's own faecal organism (E. coli) is responsible for bladder colonisation and 
breakthrough infections. This also explains why group A organisms are mostly drug resistant, while those in group B are drug sensitive.

We conclude that clean intermittent catheterisation is a safe and effective method of bladder drainage both in acute spinal trauma and established neuropathic bladder patients. Infective complications are accept- ably low and do not warrant any prophylactic antibacterial therapy. The threat of the emergence of resistant organism, unwanted side effects and the inappropriate cost of antibiotics discourages their routine use in asymptomatic patients. However, clinical infections have to be recognized separately from positive urine cultures and treated accordingly.

\section{References}

1 Bors E (1967) Intermittent catheterization in paraplegic patients. Urol Int 22: 236-249.

2 Guttmann L, Frankel H (1966) The value of intermittent cathererization in early management of traumatic paraplegia and tetraplegia. Paraplegia 4: 63-84.

3 Ott R, Rosier AB (1971) The importance of intermittent catheterization in bladder re-education of acute traumatic spinal cord lesions. In: Proc Eighteenth Vet Admin Spinal Cord Injury Conf 18: 139-148.

4 Firlet CS, Canning JR, Lloyd FA, Cross RR, Brewer RJ (1975) Experience with intermittent catheterization in chronic spinal cord injury patients. J Urol 114: 234-236.

5 Lapides J, Diokno AC, Silber SJ, Lowe BS (1972) Clean, intermittent self-catheterization in the treatment of urinary tract disease. J Urol 107: 458-461.

6 Maynard FM, Diokno AC (1984) Urinary infection and complications during clean intermittent catheterization following spinal cord injury. J Urol 132: 943-946.

7 Krebs M, Halvorsen RB, Fishman IJ, Santoz-Menduza N (1984) Prevention of urinary tract infection during intermittent catheterization. J Urol 131: 82-85.

8 Maynard FM, Diokno AC (1982) Clean intermittent catheterization for spinal cord injury patients. J Urol 128: 477-480.

9 Wyndaele JJ, Taeye De N (1990) Early intermittent self-catheterization after spinal cord injury. Paraplegia 28: 76-80.

10 Mohler JL, Cowen DL, Flanigana RC (1987) Suppression and treatment of urinary tract infection in patients with an intermittently catheterized neurogenic bladder. J Urol 138: 336-340.

11 Dean LS (1986) Suppressive antibacterial therapy in the prevention of urinary tract infection in spinal cord injury patients. Paraplegia 24: 60 .

12 Pearman JW (1971) Prevention of urinary tract infection following spinal cord injury. Paraplegia 9: 95-104.

13 Donovan WH, Stolov WC, Clowers DE, Clowers MR (1978) Bacteriuria during intermittent catheterization following spinal cord injury. Arch Phys Med Rehabil 59: 351-357.

14 Stickler DJ, Chawla JC (1988) An appraisal of antibiotic policies for urinary tract infections in patients with spinal cord injuries undergoing long-term intermittent catheterization. Paraplegia 26: 215-225. 